Pacific

Journal of

Mathematics

\title{
IDEAL DECOMPOSITIONS OF A TERNARY RING OF OPERATORS WITH PREDUAL
}

\author{
MASAYOSHI KANEDA
}




\title{
IDEAL DECOMPOSITIONS OF A TERNARY RING OF OPERATORS WITH PREDUAL
}

\author{
MASAYOSHI KANEDA
}

\begin{abstract}
We show that any TRO (ternary ring of operators) with predual can be decomposed into the direct sum of a two-sided ideal, a left ideal, and a right ideal in some von Neumann algebra using an extreme point of the unit ball of the TRO.
\end{abstract}

Recall that an operator space $X$ is called a triple system or a ternary ring of operators (TRO for short) if there exists a complete isometry $\iota$ from $X$ into a $C^{*}$-algebra such that $\iota(x) \iota(y)^{*} \iota(z) \in \iota(X)$ for all $x, y, z \in X$. Our main result is that any TRO with predual can be decomposed into the direct sum of a two-sided ideal, a left ideal, and a right ideal in some von Neumann algebra:

Theorem. Let $X$ be a TRO which is also a dual Banach space. Then $X$ can be decomposed into the direct sum of TROs $X_{T}, X_{L}$, and $X_{R}$,

$$
X=X_{T} \stackrel{\infty}{\oplus} X_{L} \stackrel{\infty}{\oplus} X_{R},
$$

so that there is a complete isometry ( from $X$ into a von Neumann algebra in which $\iota\left(X_{T}\right), \iota\left(X_{L}\right)$, and $\iota\left(X_{R}\right)$ are a weak*-closed two-sided, left, and right ideal, respectively, and

$$
\iota(X)=\iota\left(X_{T}\right) \stackrel{\infty}{\oplus} \iota\left(X_{L}\right) \stackrel{\infty}{\oplus} \iota\left(X_{R}\right) .
$$

In the special case that the TRO is finite-dimensional, the decomposition is into a direct sum of rectangular matrices, as first proved essentially by R. R. Smith [2000]. In the Appendix we give a short proof of that result. The following lemma is a version of Kadison's theorem [1951, Theorem 1] as found in [Pedersen 1979, Proposition 1.4.8] or [Sakai 1971, Proposition 1.6.5]. Together with the idea of embedding an off-diagonal corner into a diagonal corner developed in [Blecher and Kaneda 2004, Section 2] (see also [Kaneda 2003, Section 2.2]), it plays a key role in the proof of our theorem.

The author was supported by a research fund from Department of Mathematics, University of California, Irvine.

MSC2010: primary 46L45, 47L20; secondary 46L07, 47L25, 47L50, 47L07.

Keywords: extreme points, dual operator spaces, von Neumann algebras, ideals, ternary rings of operators. 
Lemma (Kadison's theorem). Let $\mathcal{A}$ be a $C^{*}$-algebra, and let $p, q$ be orthogonal projections in $\mathcal{A}$. Then an element $x \in p \mathcal{A} q$ is an extreme point of $\operatorname{Ball}(p \mathcal{A} q)$ if and only if $\left(p-x x^{*}\right) \mathcal{A}\left(q-x^{*} x\right)=\{0\}$. In this case, $x$ is a partial isometry.

Proof of the Theorem. By [Effros et al. 2001, Theorem 2.6], we may regard $X$ as a weak*-closed subspace of $\mathbb{B}(\mathcal{K}, \mathcal{H})$ for some Hilbert spaces $\mathcal{H}$ and $\mathcal{K}$ such that $X X^{*} X \subset X$. We may assume that $[X \mathcal{K}]=\mathcal{H}$ and $\left[X^{*} \mathcal{H}\right]=\mathcal{K}$. We also identify $\mathbb{B}(\mathcal{K}, \mathcal{H})$ with the $(1,2)$-corner of $\mathbb{B}(\mathcal{H} \oplus \mathcal{K})$, and let $1_{\mathcal{H}} \in \mathbb{B}(\mathcal{H} \oplus \mathcal{K})$ and $1_{\mathcal{K}} \in \mathbb{B}(\mathcal{H} \oplus \mathcal{K})$ denote the orthogonal projections on $\mathcal{H}$ and $\mathcal{K}$. Then

$$
\mathcal{L}(X):=\left[\begin{array}{cc}
\overline{X X^{*}} \mathrm{w}^{*} & X \\
X^{*} & \bar{X}^{*} \mathrm{w}^{*}
\end{array}\right]
$$

is the linking von Neumann algebra, $1_{\mathcal{H}}, 1_{\mathcal{K}} \in \mathcal{L}(X)$, and $X=1_{\mathcal{H}} \mathcal{L}(X) 1_{\mathcal{K}}$. Since $\operatorname{Ball}(X)$ is weak*-closed in $\mathbb{B}(\mathcal{K}, \mathcal{H})$, there is an extreme point $e \in \operatorname{Ball}(X)$. By Kadison's theorem above,

$$
\left(1_{\mathcal{H}}-e e^{*}\right) X\left(1_{\mathcal{K}}-e^{*} e\right)=\{0\},
$$

and $e$ is a partial isometry. Let $p \in \overline{X\left(1_{\mathcal{K}}-e^{*} e\right) X^{*}}{ }^{\mathrm{w}^{*}}$ and $q \in \overline{X^{*}\left(1_{\mathcal{H}}-e e^{*}\right) X} \mathrm{w}^{*}$ be the identities of these two von Neumann algebras. Then by the adjoint of (1), it follows that

$$
\begin{gathered}
p X q=\{0\}, \\
p=p e e^{*}=e e^{*} p=p e e^{*} p \quad \text { and } q=e^{*} e q=q e^{*} e=q e^{*} e q .
\end{gathered}
$$

Noting that $p x y^{*} \in \overline{X\left(1_{\mathcal{K}}-e^{*} e\right) X^{*}}{ }^{\mathrm{w}^{*}}$ and $q x^{*} y \in{\overline{X^{*}\left(1_{\mathcal{H}}-e e^{*}\right) X}}^{\mathrm{w}^{*}}$, we also get (4) $p x y^{*}=p x y^{*} p=x y^{*} p$ and $q x^{*} y=q x^{*} y q=x^{*} y q$ for all $x, y \in X$.

Put

$$
q_{1}:=e^{*}\left(1_{\mathcal{H}}-p\right) e\left(1_{\mathcal{K}}-q\right) \text { and } \quad q_{2}:=1_{\mathcal{K}}-q-q_{1} .
$$

We claim that $q_{1}$ and $q_{2}$ are orthogonal projections. Indeed, (4) and the fact that $p e \in X$ yield

$$
\begin{aligned}
q_{1}^{*} & =\left(1_{\mathcal{K}}-q\right) e^{*}\left(1_{\mathcal{H}}-p\right) e=e^{*} e-e^{*} p e-q e^{*} e+q e^{*} p e \\
& =e^{*} e-e^{*} p e-e^{*} e q+e^{*} p e q=q_{1}
\end{aligned}
$$

and

$$
\begin{aligned}
q_{1}^{2} & =e^{*}\left(1_{\mathcal{H}}-p\right) e\left(1_{\mathcal{K}}-q\right) e^{*}\left(1_{\mathcal{H}}-p\right) e\left(1_{\mathcal{K}}-q\right)=e^{*}\left(1_{\mathcal{H}}-p\right) e q_{1}^{*}\left(1_{\mathcal{K}}-q\right) \\
& =e^{*}\left(1_{\mathcal{H}}-p\right) e q_{1}\left(1_{\mathcal{K}}-q\right)=e^{*}\left(1_{\mathcal{H}}-p\right) e e^{*}\left(1_{\mathcal{H}}-p\right) e\left(1_{\mathcal{K}}-q\right)\left(1_{\mathcal{K}}-q\right) \\
& =e^{*} e e^{*}\left(1_{\mathcal{H}}-p\right)\left(1_{\mathcal{H}}-p\right) e\left(1_{\mathcal{K}}-q\right)\left(1_{\mathcal{K}}-q\right)=e^{*}\left(1_{\mathcal{H}}-p\right) e\left(1_{\mathcal{K}}-q\right) \\
& =q_{1} .
\end{aligned}
$$


Noting that $q_{1} q=0$, we have $q_{2}^{2}=q_{2}=q_{2}^{*}$.

To see that

$$
\left(1_{\mathcal{H}}-p\right) X\left(1_{\mathcal{K}}-e^{*} e\right)=\{0\},
$$

let $\left\{u_{\alpha}\right\}$ be an approximate identity of the $C^{*}$-algebra $X^{*} X$. Then for each $x \in X$, $p x\left(1_{\mathcal{K}}-e^{*} e\right) u_{\alpha}=x\left(1_{\mathcal{K}}-e^{*} e\right) u_{\alpha}$. Taking the limit $\alpha \rightarrow \infty$ yields that

$$
p x\left(1_{\mathcal{K}}-e^{*} e\right)=x\left(1_{\mathcal{K}}-e^{*} e\right)
$$

for $x \in X$, and hence (5) holds. Similarly,

$$
\left(1_{\mathcal{H}}-e e^{*}\right) X\left(1_{\mathcal{K}}-q\right)=\{0\}
$$

also holds.

Let $x, y \in X$. Then

$$
\begin{array}{rlr}
q_{1} x^{*} y & =e^{*}\left(1_{\mathcal{H}}-p\right) e\left(1_{\mathcal{K}}-q\right) x^{*} y & \\
& =e^{*}\left(1_{\mathcal{H}}-p\right) e x^{*} y\left(1_{\mathcal{K}}-q\right) & \text { by }(4) \\
& =e^{*} e x^{*}\left(1_{\mathcal{H}}-p\right) y\left(1_{\mathcal{K}}-q\right) & \text { by }(4) \\
& =x^{*}\left(1_{\mathcal{H}}-p\right) y\left(1_{\mathcal{K}}-q\right) & \\
& =x^{*}\left(1_{\mathcal{H}}-p\right) y e^{*} e\left(1_{\mathcal{K}}-q\right) & \text { by }(5) \\
& =x^{*} y e^{*}\left(1_{\mathcal{H}}-p\right) e\left(1_{\mathcal{K}}-q\right) & \text { by }(4) \\
& =x^{*} y q_{1}, &
\end{array}
$$

and so we have

$$
q_{1} x^{*} y=x^{*} y q_{1}=q_{1} x^{*} y q_{1} \quad \text { for all } x, y \in X .
$$

Put $X_{T}:=X q_{1}, X_{L}:=X q$, and $X_{R}:=X q_{2}$. Then these are weak*-closed TROs, and $X=X_{T} \oplus X_{L} \oplus X_{R}$. Using (4) and (7) and noting that $q_{1}, q$, and $q_{2}$ are mutually disjoint, we have

$$
X_{T}^{*} X_{L}=X_{T}^{*} X_{R}=X_{L}^{*} X_{T}=X_{L}^{*} X_{R}=X_{R}^{*} X_{T}=X_{R}^{*} X_{L}=\{0\}
$$

and

$$
X^{*} X=X_{T}^{*} X_{T} \stackrel{\infty}{\oplus} X_{L}^{*} X_{L} \stackrel{\infty}{\oplus} X_{R}^{*} X_{R}
$$

This proves that $X=X_{T} \stackrel{\infty}{\oplus} X_{L} \stackrel{\infty}{\oplus} X_{R}$.

Define

$$
\iota: X \rightarrow{\overline{X X^{*}}}^{\mathrm{w}^{*}} \oplus{\overline{X^{*} X}}^{\mathrm{w}^{*}}
$$

by

$$
\iota(x):=\left(x_{T}+x_{L}\right) e^{*} \oplus e^{*} x_{R},
$$


where $x=x_{T}+x_{L}+x_{R}$ is the unique decomposition of $x \in X$ such that $x_{T} \in X_{T}$, $x_{L} \in X_{L}$, and $x_{R} \in X_{R}$. First note that $\iota\left(X_{T}\right) \cap \iota\left(X_{L}\right)=\{0\}$. Indeed, assume that $\iota\left(x_{T}\right)+\iota\left(x_{L}\right)=0$, that is, $x q_{1} e^{*}+x q e^{*}=0$. Then by multiplying both sides by $e$ on the right and using (3) and (7), we obtain that $x e^{*} e q_{1}+x q=0$. Multiplying both sides by $q$ on the right noting that $q_{1} q=0$ yields that $x q=0$, and hence $x q_{1} e^{*}=x q e^{*}=0$, that is, $\iota\left(x_{T}\right)=\iota\left(x_{L}\right)=0$. Since $\iota\left(X_{T}\right)^{*} \iota\left(X_{L}\right)=e X_{T}^{*} X_{L} e^{*}=\{0\}$ and $\iota\left(X_{L}\right)^{*} \iota\left(X_{T}\right)=e X_{L}^{*} X_{T} e^{*}=\{0\}$, we obtain

$$
\left(\iota\left(X_{T}\right) \oplus \iota\left(X_{L}\right)\right)^{*}\left(\iota\left(X_{T}\right) \oplus \iota\left(X_{L}\right)\right)=\iota\left(X_{T}\right)^{*} \iota\left(X_{T}\right) \stackrel{\infty}{\oplus} \iota\left(X_{L}\right)^{*} \iota\left(X_{L}\right)
$$

noting that $\iota\left(X_{T}\right)^{*} \iota\left(X_{T}\right)=q_{1} X_{T}^{*} X_{T} q_{1}$ and $\iota\left(X_{L}\right)^{*} \iota\left(X_{L}\right)=q X_{L}^{*} X_{L} q$. Thus $\iota(X)=$ $\iota\left(X_{T}\right) \stackrel{\infty}{\oplus} \iota\left(X_{L}\right) \stackrel{\infty}{\oplus} \iota\left(X_{R}\right)$. To show that $\iota$ is a complete isometry, it suffices to show that each of $\iota_{\mid X_{T}}, \iota_{\mid X_{L}}$, and $\iota_{\mid X_{R}}$ is a complete isometry. Since $e^{*} e q_{1}=q_{1}$,

$$
\left\|\iota\left(x_{T}\right)\right\|^{2}=\left\|\iota\left(x_{T}\right) \iota\left(x_{T}\right)^{*}\right\|=\left\|x q_{1} e^{*} e q_{1} x^{*}\right\|=\left\|x q_{1} x^{*}\right\|=\left\|x q_{1}\right\|^{2}=\left\|x_{T}\right\|^{2} .
$$

A similar calculation works at the matrix level, which concludes that $\iota_{\mid X_{T}}$ is a complete isometry. Similarly, (3) yields that $\iota_{\mid X_{L}}$ is a complete isometry.

$$
\begin{aligned}
\left\|\iota\left(x_{R}\right)\right\|^{2} & =\left\|\iota\left(x_{R}\right)^{*} \iota\left(x_{R}\right)\right\|=\left\|q_{2} x^{*} e e^{*} x q_{2}\right\|=\left\|q_{2} x^{*} e e^{*} x\left(1_{\mathcal{K}}-q-q_{1}\right)\right\| \\
& =\left\|q_{2} x^{*} x\left(1_{\mathcal{K}}-q\right)\right\|=\left\|q_{2} x^{*} x\left(1_{\mathcal{K}}-q-q_{1}\right)\right\|=\left\|q_{2} x^{*} x q_{2}\right\|=\left\|x_{R}\right\|^{2},
\end{aligned}
$$

where we used (6) and (7) as well as the fact that $q_{2} q_{1}=0$ in the fourth equality, and (7) together with the fact that $q_{2} q_{1}=0$ in the fifth equality. A similar calculation works at the matrix level, which concludes that $\iota_{\mid X_{R}}$ is a complete isometry.

By [Blecher 2001, Lemma 1.5(3)] or [Blecher and Le Merdy 2004, Theorem A.2.5(3)] for example, $\iota\left(X_{T}\right), \iota\left(X_{L}\right)$, and $\iota\left(X_{R}\right)$ are weak*-closed. Clearly, $\iota\left(X_{T}\right)$ and $\iota\left(X_{L}\right)$ are left ideals and $\iota\left(X_{R}\right)$ is a right ideal in the von Neumann algebra $\overline{X X^{*}}{ }^{\mathrm{w}^{*}} \oplus \bar{X}^{*} X^{\mathrm{w}^{*}}$. To see that $\iota\left(X_{T}\right)$ is a right ideal as well, it suffices to show that $\iota\left(X_{T}\right)^{*} \subset \iota\left(X_{T}\right)$, in which case necessarily $\iota\left(X_{T}\right)^{*}=\iota\left(X_{T}\right)$. To show this, first note that it follows from the adjoint of (6) that

$q_{1} x^{*}=e^{*}\left(1_{\mathcal{H}}-p\right) e\left(1_{\mathcal{K}}-q\right) x^{*}=e^{*}\left(1_{\mathcal{H}}-p\right) e\left(1_{\mathcal{K}}-q\right) x^{*} e e^{*}=q_{1} x^{*} e e^{*}$ for all $x \in X$.

Therefore, together with (7), we obtain

$$
\iota\left(x_{T}\right)^{*}=e q_{1} x^{*}=e q_{1} x^{*} e e^{*}=e x^{*} e q_{1} e^{*} \in X q_{1} e^{*}=\iota\left(X_{T}\right) \text { for all } x \in X .
$$

Definition. We call the decomposition $X=X_{T} \stackrel{\infty}{\oplus} X_{L} \stackrel{\infty}{\oplus} X_{R}$ obtained in the proof of Theorem the ideal decomposition of the TRO $X$ with predual with respect to an extreme point $e$ of $\operatorname{Ball}(X)$. 
Remarks. (A) The reader should distinguish ideal decompositions from Peirce decompositions in the literature of Jordan triples. In fact, a TRO can be regarded as a Jordan triple with the canonical symmetrization of the triple product. However, an ideal decomposition and a Peirce decomposition give totally different decompositions.

(B) It is also possible to define $\iota: X \rightarrow \overline{X X^{*}}{ }^{\mathrm{w}^{*}} \oplus{\overline{X^{*}}}^{\mathrm{w}^{*}}$ by

$$
\iota(x):=x_{L} e^{*} \oplus e^{*}\left(x_{R}+x_{T}\right) \text { for } x \in X .
$$

(C) Simpler expressions for $X_{T}$ and $X_{R}$ are $X_{T}=\{x-p x-x q \mid x \in X\}$ and $X_{R}=p X$, which would be more helpful in understanding what is going on in the decomposition. To see the equivalences of expressions, let $x \in X$. Then, using (4), (5), and (2), we have

$$
\begin{aligned}
x_{T}: & =x q_{1}=x e^{*}\left(1_{\mathcal{H}}-p\right) e\left(1_{\mathcal{K}}-q\right)=\left(1_{\mathcal{H}}-p\right) x e^{*} e\left(1_{\mathcal{K}}-q\right) \\
& =\left(1_{\mathcal{H}}-p\right) x\left(1_{\mathcal{K}}-q\right)=x-p x-x q .
\end{aligned}
$$

Accordingly, it follows that

$x_{R}:=x q_{2}=x\left(1_{\mathcal{K}}-q-q_{1}\right)=x\left(1_{\mathcal{K}}-q\right)-x q_{1}=x\left(1_{\mathcal{K}}-q\right)-(x-p x-x q)=p x$.

(D) The ideal decomposition highly depends on the extreme point chosen. Indeed, let $X$ be a von Neumann algebra, $u \in X$ be a unitary element, and $w \in X$ be an isometry which is not unitary. Then the ideal decomposition with respect to $u$ is just $X=X_{T}$, while the one with respect to $w$ is $X=X_{T} \stackrel{\infty}{\oplus} X_{L}$.

\section{Appendix: A short proof of Smith's result}

The following theorem was proved in [Smith 2000] (also see [Effros and Ruan 2000, Lemma 6.1.7 and Corollary 6.1.8]). We observed it independently in 2000, together with Corollary A.2. Since these results are a special case of this paper's Theorem, and our proof is short enough to understand the essence of the results transparently, it seems worthwhile to present them here. The key to the shortness of the proof is the obvious fact that if a TRO $X$ is finite-dimensional, then so are the $C^{*}$-algebras $X X^{*}$ and $X^{*} X$.

Theorem A.1 [Smith 2000]. If $X$ is a finite-dimensional TRO, then there exist a finite-dimensional $C^{*}$-algebra $\mathcal{A}$ and an orthogonal projection $p \in \mathcal{A}$ such that $X \cong p \mathcal{A} p^{\perp}$ completely isometrically.

Proof. Let $X \subset \mathbb{B}(\mathcal{K}, \mathcal{H})$ be a finite-dimensional TRO and $\left\{x_{1}, \ldots, x_{n}\right\} \subset X$ be its base. We may assume that $[X \mathcal{K}]=\mathcal{H}$ and $\left[X^{*} \mathcal{H}\right]=\mathcal{K}$. Then the $C^{*}$-algebra $X X^{*}:=\operatorname{span}\left\{x y^{*} \mid x, y \in X\right\}$ is equal to the $\operatorname{set} \operatorname{span}\left\{x_{i} x_{j}^{*} \mid 1 \leq i, j \leq n\right\}$, and the latter is obviously a finite-dimensional vector space. Similarly, $X^{*} X:=\operatorname{span}\left\{x^{*} y \mid\right.$ 
$x, y \in X\}$ is a finite-dimensional $C^{*}$-algebra. Let $\mathcal{L}(X)$ be the linking $C^{*}$-algebra for $X$, that is,

$$
\mathcal{L}(X):=\left[\begin{array}{cc}
X X^{*} & X \\
X^{*} & X^{*} X
\end{array}\right](\subset B(\mathcal{H} \oplus \mathcal{K})) .
$$

Let $e, f$ be the identities of the $C^{*}$-algebras $X X^{*}$ and $X^{*} X$, respectively, and let

$$
p:=\left[\begin{array}{ll}
e & 0 \\
0 & 0
\end{array}\right] \in \mathcal{L}(X) .
$$

Then

$$
p^{\perp}=\left[\begin{array}{ll}
0 & 0 \\
0 & f
\end{array}\right]
$$

and $X \cong p \mathcal{L}(X) p^{\perp}$ completely isometrically.

Corollary A.2. A finite-dimensional TRO is completely isometric to the direct sum of rectangular matrices: $\mathbb{M}_{l_{1}, k_{1}}(\mathbb{C}) \stackrel{\infty}{\oplus} \cdots \stackrel{\infty}{\oplus} \mathbb{M}_{l_{m}, k_{m}}(\mathbb{C})$.

Proof. Let $X$ be a finite-dimensional TRO. By Theorem A.1, we may assume that $X=p\left(\bigoplus_{i=1}^{m} \mathbb{M}_{n_{i}}(\mathbb{C})\right) p^{\perp}$, where $p$ is an orthogonal projection in $\bigoplus_{i=1}^{m} \mathbb{M}_{n_{i}}(\mathbb{C})$. For each $1 \leq i \leq m$, let us denote by $1_{i}$ the identity of $\mathbb{M}_{n_{i}}(\mathbb{C})$ which is identified with an element of $\bigoplus_{i=1}^{m} \mathbb{M}_{n_{i}}(\mathbb{C})$ in the obvious way, and let $p_{i}:=p 1_{i}$. Then $X=\bigoplus_{i=1}^{m} p_{i} \mathbb{M}_{n_{i}}(\mathbb{C}) p_{i}^{\perp}$. By a unitary transform which is a complete isometry, we may assume that

$$
p_{i}=\operatorname{diag}\{\overbrace{1, \ldots, 1}^{l_{i} \text { times }}, \overbrace{0, \ldots, 0}^{\left(n_{i}-l_{i}\right) \text { times }}\} \text { and } \quad p_{i}^{\perp}=\operatorname{diag}\{\overbrace{0, \ldots, 0}^{l_{i} \text { times }}, \overbrace{1, \ldots, 1}^{\left(n_{i}-l_{i}\right) \text { times }}\}
$$

for each $1 \leq i \leq m$.

\section{References}

[Blecher 2001] D. P. Blecher, "Multipliers and dual operator algebras", J. Funct. Anal. 183:2 (2001), 498-525. MR 2003c:46075 Zbl 1027.47077

[Blecher and Kaneda 2004] D. P. Blecher and M. Kaneda, "The ideal envelope of an operator algebra", Proc. Amer. Math. Soc. 132:7 (2004), 2103-2113. MR 2005a:46106 Zbl 1046.46042

[Blecher and Le Merdy 2004] D. P. Blecher and C. Le Merdy, Operator algebras and their modules: an operator space approach, London Mathematical Society Monographs, New Series 30, Clarendon Press/Oxford University Press, Oxford, 2004. MR 2006a:46070 Zbl 1061.47002

[Effros and Ruan 2000] E. G. Effros and Z.-J. Ruan, Operator spaces, London Mathematical Society Monographs, New Series 23, Clarendon Press/Oxford University Press, New York, 2000. MR 2002a:46082 Zbl 0969.46002

[Effros et al. 2001] E. G. Effros, N. Ozawa, and Z.-J. Ruan, "On injectivity and nuclearity for operator spaces”, Duke Math. J. 110:3 (2001), 489-522. MR 2002k:46151 Zbl 1010.46060

[Kadison 1951] R. V. Kadison, "Isometries of operator algebras", Ann. Math. (2) 54 (1951), 325-338. MR 13,256a Zbl 0045.06201 
[Kaneda 2003] M. Kaneda, Multipliers and algebrizations of operator spaces, $\mathrm{PhD}$ thesis, University of Houston, 2003, Available at http://search.proquest.com/docview/305326901. MR 2704919

[Pedersen 1979] G. K. Pedersen, $C^{*}$-algebras and their automorphism groups, London Mathematical Society Monographs 14, Academic Press, London, 1979. MR 81e:46037 Zbl 0416.46043

[Sakai 1971] S. Sakai, $C^{*}$-algebras and $W^{*}$-algebras, Ergebnisse der Mathematik und ihrer Grenzgebiete 60, Springer, New York, 1971. MR 56 \#1082 Zbl 0219.46042

[Smith 2000] R. R. Smith, "Finite dimensional injective operator spaces", Proc. Amer. Math. Soc. 128:11 (2000), 3461-3462. MR 2001d:46087 Zbl 0962.46042

Received September 30, 2012.

MASAYOSHI KANEDA

DEPARTMENT OF MATHEMATICS

UNIVERSITY OF CALIFORNIA, IRVINE

340 ROWLAND HALL

IRVINE, CA 92697-3875

UNITED STATES

Current address:

DEPARTMENT OF MATHEMATICS

SCHOOL OF SCIENCE AND TECHNOLOGY

NAZARBAYEV UNIVERSITY

53 Kabanbay Batyr AVEnUE

ASTANA 010000

KAZAKHSTAN

mkaneda@uci.edu

http://sst.nu.edu.kz/sst/Academics/departments/Mathematics/Mathematics_People/MasayoshiKaneda 


\title{
PACIFIC JOURNAL OF MATHEMATICS
}

\author{
msp.org/pjm
}

Founded in 1951 by E. F. Beckenbach (1906-1982) and F. Wolf (1904-1989)

\section{EDITORS}

V. S. Varadarajan (Managing Editor)

Department of Mathematics

University of California

Los Angeles, CA 90095-1555

pacific@math.ucla.edu

Paul Balmer

Department of Mathematics

University of California

Los Angeles, CA 90095-1555

balmer@math.ucla.edu

Daryl Cooper

Department of Mathematics

University of California

Santa Barbara, CA 93106-3080 cooper@math.ucsb.edu

Jiang-Hua Lu

Department of Mathematics

The University of Hong Kong

Pokfulam Rd., Hong Kong jhlu@maths.hku.hk
Don Blasius

Department of Mathematics University of California

Los Angeles, CA 90095-1555

blasius@math.ucla.edu

Robert Finn

Department of Mathematics Stanford University

Stanford, CA 94305-2125

finn@math.stanford.edu

Sorin Popa

Department of Mathematics

University of California

Los Angeles, CA 90095-1555 popa@math.ucla.edu

Paul Yang

Department of Mathematics Princeton University

Princeton NJ 08544-1000

yang@math.princeton.edu

\section{PRODUCTION}

Silvio Levy, Scientific Editor, production@msp.org

\section{SUPPORTING INSTITUTIONS}

ACADEMIA SINICA, TAIPEI

CALIFORNIA INST. OF TECHNOLOGY

INST. DE MATEMÁTICA PURA E APLICADA

KEIO UNIVERSITY

MATH. SCIENCES RESEARCH INSTITUTE

NEW MEXICO STATE UNIV.

OREGON STATE UNIV.

\author{
STANFORD UNIVERSITY \\ UNIV. OF BRITISH COLUMBIA \\ UNIV. OF CALIFORNIA, BERKELEY \\ UNIV. OF CALIFORNIA, DAVIS \\ UNIV. OF CALIFORNIA, LOS ANGELES \\ UNIV. OF CALIFORNIA, RIVERSIDE \\ UNIV. OF CALIFORNIA, SAN DIEGO \\ UNIV. OF CALIF., SANTA BARBARA
}

\author{
Vyjayanthi Chari \\ Department of Mathematics \\ University of California \\ Riverside, CA 92521-0135 \\ chari@math.ucr.edu \\ Kefeng Liu \\ Department of Mathematics \\ University of California \\ Los Angeles, CA 90095-1555 \\ liu@math.ucla.edu \\ Jie Qing \\ Department of Mathematics \\ University of California \\ Santa Cruz, CA 95064 \\ qing@cats.ucsc.edu
}

These supporting institutions contribute to the cost of publication of this Journal, but they are not owners or publishers and have no responsibility for its contents or policies.

See inside back cover or msp.org/pjm for submission instructions.

The subscription price for 2013 is US \$400/year for the electronic version, and \$485/year for print and electronic.

Subscriptions, requests for back issues and changes of subscribers address should be sent to Pacific Journal of Mathematics, P.O. Box 4163, Berkeley, CA 94704-0163, U.S.A. The Pacific Journal of Mathematics is indexed by Mathematical Reviews, Zentralblatt MATH, PASCAL CNRS Index, Referativnyi Zhurnal, Current Mathematical Publications and Web of Knowledge (Science Citation Index).

The Pacific Journal of Mathematics (ISSN 0030-8730) at the University of California, c/o Department of Mathematics, 798 Evans Hall \#3840, Berkeley, CA 94720-3840, is published twelve times a year. Periodical rate postage paid at Berkeley, CA 94704, and additional mailing offices. POSTMASTER: send address changes to Pacific Journal of Mathematics, P.O. Box 4163, Berkeley, CA 94704-0163.

PJM peer review and production are managed by EditFLOW ${ }^{\circledR}$ from Mathematical Sciences Publishers.

\section{PUBLISHED BY}

mathematical sciences publishers

nonprofit scientific publishing

http://msp.org/

(C) 2013 Mathematical Sciences Publishers 


\section{PACIFIC JOURNAL OF MATHEMATICS}

Volume $266 \quad$ No. $2 \quad$ December 2013

Rate of attraction for a semilinear wave equation with variable coefficients and

critical nonlinearities

FÁGNER DiAs ARARUNA and FLANK DAVID MORAIS BEZERRA

The Brin-Thompson groups $s V$ are of type $\mathrm{F}_{\infty}$

Martin G. Fluch, Marco Marschler, Stefan Witzel and

MATTHEW C. B. ZAREMSKY

Ideal decompositions of a ternary ring of operators with predual

MASAYOSHI KANEDA

A study of real hypersurfaces with Ricci operators in 2-dimensional complex space 305 forms

Dong Ho Lim, WoOn Ha SoHn and Hyunjung Song

On commensurability of fibrations on a hyperbolic 3-manifold

HideTOSHI MASAI

Multiplicative Dirac structures

CRISTIÁN ORTIZ

On the finite generation of a family of Ext modules

TONY J. PuthenPuRAKAL

Index formulae for Stark units and their solutions

XAVIER-FranÇOIS ROBLOT

The short time asymptotics of Nash entropy

GUOYI XU

Several splitting criteria for vector bundles and reflexive sheaves

STEPHEN S.-T. YAU and FEI YE

The minimal volume orientable hyperbolic 3-manifold with 4 cusps

KEN'ICHI YOSHIDA

On the Witten rigidity theorem for string ${ }^{c}$ manifolds

JIANQING YU and BO LIU 